\title{
Comparison of Cryoballoon and Contact Force-Sensing Radiofrequency Ablation for Persistent Atrial Fibrillation in Clinical Practice
}

\author{
Atsushi Kobori, MD, PhD; Yasuhiro Sasaki, MD; Misun Pak, MD, PhD; Masahiro Ishikura, MD; \\ Ryosuke Murai, MD; Taiji Okada, MD, PhD; Toshiaki Toyota, MD, PhD; \\ Tomohiko Taniguchi, MD, PhD; Kitae Kim, MD, PhD; Natsuhiko Ehara, MD, PhD; \\ Makoto Kinoshita, MD, PhD; Yasuki Kihara, MD, PhD; Yutaka Furukawa, MD, PhD
}

Background: Outcomes of cryoballoon ablation for persistent atrial fibrillation (AF) are unclear, especially in Japanese patients, so the effectiveness and safety of cryoballoon ablation in clinical practice were retrospectively compared with those of contact forcesensing radiofrequency (CFRF) ablation including the high-power protocol.

Methods and Results: Consecutive patients with persistent AF were reviewed, and 253 and 265 patients who underwent cryoballoon and CFRF ablation, respectively, were enrolled. The primary endpoint was atrial arrhythmia recurrence. The secondary endpoints were periprocedural complications and repeat ablation. The rate of additional left atrial (LA) ablation after pulmonary vein isolation (PVI) was similar between groups $(68.8 \%$ cryoballoon vs. $74.0 \% \mathrm{CFRF}, \mathrm{P}=0.19)$. Freedom from atrial arrhythmia recurrence was comparable between groups over a follow-up of $25.5 \pm 12.5$ months (72.3\% cryoballoon vs. $69.8 \%$ CFRF; adjusted hazard ratio (HR) $0.85,95 \%$ confidence interval $(\mathrm{Cl}) 0.59-1.21, \mathrm{P}=0.36)$. Outcomes were similar in the subgroups of $\mathrm{PVI}$ alone and $\mathrm{PVI}$ plus additional LA ablation. LA posterior wall isolation, absence of defragmentation, and low creatine clearance, but not catheter selection, were associated with the primary endpoint. Periprocedural complications (adjusted HR 0.73, 95\% $\mathrm{Cl} 0.34-1.54, \mathrm{P}=0.41$ ) and repeat ablation (adjusted HR 1.11, 95\% Cl 0.71-1.74, $\mathrm{P}=0.64$ ) were similar for both procedures.

Conclusions: Cryoballoon ablation for persistent AF in Japanese clinical practice had acceptable outcomes comparable to those of advanced CFRF ablation.

Key Words: Catheter ablation; Contact force-sensing radiofrequency ablation; Cryoballoon; Persistent atrial fibrillation

I $\mathrm{n}$ Japan, the number of patients with atrial fibrillation (AF) is increasing with the aging of society, and it is expected that the use of catheter ablation (CA) in the treatment of AF will increase. ${ }^{1}$ The basic strategy of AF ablation is pulmonary vein isolation (PVI), and balloon catheters designed to achieve more efficient PVI have recently become available.,2,3 Many balloon catheters provide good outcomes as specific PVI devices; however, until recently, their indication was limited to paroxysmal AF in Japan. ${ }^{4}$

In recent years, it has been reported that a sufficient therapeutic effect may be obtained with PVI alone even in persistent AF, and the cryoballoon has become available for the treatment of patients with persistent AF. -11 $^{-11}$ Therefore, use of the cryoballoon is expected to expand the treatment modalities for persistent AF in clinical settings as an alternative device. Compared with radiofrequency (RF)
CA, PVI using a cryoballoon is noted for the short procedure time, high safety, and high durability. ${ }^{12-15}$ However, with the advent of contact force-sensing and high-power short-duration protocol treatment guided by the lesion formation index, RF catheters have recorded shorter procedure times and improved safety. ${ }^{16}$ Although rapid technological innovation seems to have further enhanced the advantages of catheters, only a few studies have directly compared the usefulness of both types of CA for persistent AF in real-world settings. ${ }^{17}$

In addition, many challenges beyond PVI have been reported to result in better outcomes in some patients; however, the treatment strategy for persistent AF is still not established. ${ }^{18}$ In fact, there are many cases in which additional treatment beyond PVI is performed based on the experience of the operators in clinical practice. On this basis, the real usefulness of cryoballoon ablation for per-

Received July 7, 2021; revised manuscript received August 1, 2021; accepted August 8, 2021; J-STAGE Advance Publication released online September 25, 2021 Time for primary review: 12 days

Department of Cardiology, Kobe City Medical Center General Hospital, Kobe, Japan

Mailing address: Atsushi Kobori, MD, PhD, Department of Cardiology, Kobe City Medical Center General Hospital, 2-1-1 Minatojima-minamimachi, Chuou-ku, Kobe 650-0047, Japan. E-mail: kobori40@gmail.com

All rights are reserved to the Japanese Circulation Society. For permissions, please e-mail: cj@j-circ.or.jp

ISSN-1346-9843 


\begin{tabular}{lccc} 
Table 1. Baseline Patient Demographics & Cryoballoon & CFRF & P value \\
& $(\mathbf{n = 2 5 3 )}$ & $(\mathbf{n = 2 6 5 )}$ & 0.33 \\
Age, years & $70.0 \pm 9.6$ & $70.7 \pm 9.1$ & 0.028 \\
Male sex, \% & 78.3 & 69.8 & 0.76 \\
Disease periods, months & $7[5.5,22]$ & $7[5,18]$ & 0.005 \\
Duration of AF, months & $5[4,7]$ & $6[4,7]$ & 0.34 \\
CHADS, score & $1[1,2]$ & $1[1,2]$ & 0.90 \\
No. of ineffective antiarrhythmic drugs & $1[1,1.5]$ & $1[1,2]$ & 0.07 \\
No baseline disease, \% & 28.1 & 21.1 & 0.21 \\
Hypertension, \% & 54.9 & 60.4 & 0.028 \\
Heart failure, \% & 24.2 & 32.9 & 0.47 \\
Cardiomyopathy, \% & 4.0 & 4.0 & 0.045 \\
Valvular heart disease, \% & 3.6 & 7.6 & 0.08 \\
Renal dysfunction, \% $<\mathrm{n}$ of $\mathrm{HD}>$ & $4.0,<5>$ & $7.6,<5>$ & 0.73 \\
Respiratory disease, \% & 5.8 & 6.5 & 0.005 \\
LVEF, \% & $58.9 \pm 7.9$ & $56.7 \pm 9.6$ & 0.003 \\
Left atrial diameter, mm & $41.4 \pm 5.2$ & $42.9 \pm 5.9$ & 0.11 \\
Left atrial volume index, $\mathrm{mL} / \mathrm{m}{ }^{2}$ & $42.1 \pm 20.0$ & $37.7 \pm 11.2$ & 0.07 \\
Left appendage flow velocity, $\mathrm{cm} / \mathrm{s}$ & $38.6 \pm 19.9$ & $35.2 \pm 22.5$ & 0.30 \\
Left common pulmonary vein, $\mathrm{n}$ & 6 & 34 & 0.73 \\
Right middle pulmonary vein, $\mathrm{n}$ & 14 & 20 & \\
\hline
\end{tabular}

Continuous variables are shown as mean $\pm \mathrm{SD}$ or as median [interquartile] values. $\mathrm{AF}$, atrial fibrillation; CFRF, contact force-sensing radiofrequency catheter; HD, patients on hemodialysis; LVEF, left ventricular ejection fraction; $\mathrm{n}$, number of patients.

sistent $\mathrm{AF}$ in current clinical practice will be clarified by comparing it with the contact force-sensing RF (CFRF) ablation as a reliable common device.

\section{Methods}

\section{Study Patients}

This study screened consecutive patients who underwent their first CA using the cryoballoon or CFRF because of drug-resistant persistent AF between September 2014 and May 2020. Patients with long-lasting AF ( $>1$ year), enlarged left atrium (LA diameter $>60 \mathrm{~mm}$ ), severe valvular disease, and unstable hemodynamic condition were excluded. Because the cryoballoon is non-compliant, it is difficult to occlude a PV that has been deformed into a teardrop or severe oval shape due to vertebral body compression or to dilate the balloon in a LA that is considerably less than the balloon diameter; therefore, such patients are less indicated for cryoballoon PVI. ${ }^{4}$ Eligible patients for cryoballoon ablation were selected based on preprocedural computed tomography (CT) images, and patients who had a severely thin LA or deformed PV were excluded. PVI was performed in all cases using a cryoballoon (cryoballoon group) or RF catheter (CFRF group). Patients requiring additional ablation lesions in the LA underwent treatment at the operator's discretion. The study was approved by the Institutional Review Board of Kobe City Medical Center General Hospital, and an opt-out system was used to obtain patients' consent for the use of their clinical data for research purposes. This study was conducted according to the principles of the Declaration of Helsinki.

\section{Ablation}

Periprocedural Management and Electrophysiological
Study All patients received oral anticoagulation medications between 1 month preoperatively and 6 months postoperatively. All anticoagulation medications, except warfarin, were skipped once before the procedure and immediately restarted after the procedure. All antiarrhythmic medications were discontinued for at least 5 half-lives before the procedure. An activated clotting time $>300$ s was maintained by continuous heparin infusion during the procedure. Dexmedetomidine and fentanyl were used to achieve conscious sedation in patients. Multipolar catheters with internal cardioversion (BeeAT, Japan-Life-Line, Tokyo, Japan) were placed in the coronary sinus through the internal jugular vein, and a deflectable multipolar catheter was placed in the right ventricle or right atrium through the right femoral vein. Transseptal puncture was performed with an RF needle (Baylis Medical, Montreal, QC, Canada) under intracardiac echocardiographic guidance, and 1 or 2 sheaths were introduced into the LA. Three-dimensional (3D) voltage or complex fractionated atrial electrogram (CFAE) maps of the LA and PVs before PVI were obtained with an EnSite Precision mapping system (Abbott, St. Paul, MN, USA) in the cryoballoon group or the CARTO3 system (version 6 or 7; Biosense Webster Inc., Irvine, CA, USA) in the CFRF group. The preprocedural CT image of the LA was integrated with the 3D electroanatomical map. Esophageal luminal temperature was monitored using a temperature-sensing probe (Esophastar, Japan-Life-Line, cryoballoon group; SensiTherm Multi, Abbott, CFRF group).

To confirm the completion of PVI, the bidirectional block of paced or self-activated PV electrograms was assessed using a 10 or 20 multipolar circular catheter after the ablation of all PVs. Almost all patients underwent ablation at the cavotricuspid isthmus line using an RF catheter. If non-PV foci, including the superior vena cava 


\begin{tabular}{|c|c|c|c|}
\hline & $\begin{array}{c}\text { Cryoballoon } \\
(n=253)\end{array}$ & $\begin{array}{c}\text { CFRF } \\
(n=265)\end{array}$ & $P$ value \\
\hline PVI completion, \% & 100 & 100 & \\
\hline Touch-up PVI by radiofrequency ablation, $\mathrm{n}(\%)$ & $10(3.9)$ & - & \\
\hline LA roof line, success $(\%) /$ performed $(\%)$ & $145(92.4) / 157(62.1)$ & $145(75.1) / 193(72.8)$ & $<0.0001 / 0.009$ \\
\hline LA posterior line, success (\%)/performed (\%) & $108(89.3) / 121(47.8)$ & $175(92.1) / 190(71.7)$ & $0.39 /<0.001$ \\
\hline LA posterior wall isolation, success (\%)/performed (\%) & $101(84.2) / 120(47.4)$ & $132(70.2) / 188(70.9)$ & $0.005 /<0.0001$ \\
\hline Mitral isthmus line, success (\%)/performed (\%) & $6(75.0) / 8(3.2)$ & $27(84.4) / 32(12.1)$ & $0.55 /<0.0001$ \\
\hline Non-PV foci/AT, success (\%)/performed (\%) & $9(100) / 9(3.5)$ & $16(76.2) / 21(7.9)$ & $0.05 /<0.05$ \\
\hline Defragmentation, n (\%) & $93(36.7)$ & $154(58.1)$ & $<0.0001$ \\
\hline SVC isolation, $\mathrm{n}(\%)$ & $2(0.8)$ & $2(0.8)$ & 0.88 \\
\hline CTI line, $n(\%)$ & $243(96.0)$ & $251(96.7)$ & 0.92 \\
\hline \multicolumn{4}{|l|}{ Time } \\
\hline $\mathrm{PVI}, \min$ & $34.5 \pm 16.4$ & $72.9 \pm 32.7$ & $<0.0001$ \\
\hline Dwelling in LA, min & $90.5 \pm 33.7$ & $126.0 \pm 39.0$ & $<0.0001$ \\
\hline Total procedure, $\min$ & $163.7 \pm 40.5$ & $207.3 \pm 47.6$ & $<0.0001$ \\
\hline Fluoroscopy, min & $37.9 \pm 1.0$ & $20.1 \pm 1.0$ & $<0.0001$ \\
\hline Fluid balance, $\mathrm{mL}$ & $+582.9 \pm 449$ & $+1,219.6 \pm 613$ & $<0.0001$ \\
\hline Periprocedural adverse events & 14 & 18 & 0.55 \\
\hline Bradycardia, $\mathrm{n}$ & 0 & 1 & 0.25 \\
\hline Cardiac tamponade/pericardial effusion, $n / n$ & $1 / 1$ & $2 / 1$ & 0.69 \\
\hline Coronary artery spasm, $\mathrm{n}$ & 2 & 1 & 0.53 \\
\hline Cerebrovascular event, $\mathrm{n}$ & 0 & 1 & 0.25 \\
\hline Gastrointestinal disorder, $\mathrm{n}$ & 0 & 1 & 0.25 \\
\hline Heart failure worsening, $n$ & 1 & 3 & 0.33 \\
\hline Hematoma/bleeding, $n$ & 3 & 5 & 0.52 \\
\hline Pericarditis, $\mathrm{n}$ & 0 & 2 & 0.10 \\
\hline Phrenic nerve palsy, transient/at-discharge, $n / n$ & $3 / 2$ & $1 / 0$ & 0.08 \\
\hline PV stenosis, $n$ & 0 & 0 & 0.10 \\
\hline Thrombosis, peripheral/pulmonary, $\mathrm{n} / \mathrm{n}$ & $1 / 0$ & $0 / 0$ & 0.23 \\
\hline
\end{tabular}

Continuous variables are shown as mean \pm standard deviation. AT, atrial tachycardia; CTI, cavotricuspid isthmus; LA, left atrium; PV, pulmonary vein; PVI, pulmonary vein isolation; SVC, superior vena cava. Other abbreviations as in Table 1.

were detected, superior vena cava isolation or focus ablation was performed using an RF catheter. On the other hand, additional ablations, including linear ablation at the LA roof, LA posterior wall, and mitral isthmus, and defragmentation for CFAE were performed based on the operator's discretion. We performed the provocation test for AF by rapid and decrement pacing at the right atrium or coronary sinus with an infusion of $20-40 \mu \mathrm{g}$ of isoproterenol, and any continuous AF was terminated by internal cardioversion. A figure-of-eight suture was used before all vein sheath extractions, and the patients remained in bed for $6 \mathrm{~h}$ after the procedure.

Cryoballoon Ablation The cryoballoon ablation procedure was performed as previously described. ${ }^{4}$ Briefly, after voltage mapping of the LA and PV, a 15-Fr steerable sheath (FlexCath Advance, Medtronic Inc., MN, USA) was placed. A $28-\mathrm{mm}$ second- or fourth-generation cryoballoon (ArcticFront Advance, or ArcticFront Advance Pro, Medtronic Inc.) was inflated at the orifice of each PV and anchored using a 20-mm circular mapping wire catheter with 10-pole electrodes (Achieve, Medtronic Inc.). Optimal occlusion was confirmed using the pooling and leakage maneuver (proximal seal technique). The singlefreezing protocol (180s) was initially applied to each PV in the following order: left superior, left inferior, right inferior, and right superior. When the esophageal luminal temperature reached $<15^{\circ} \mathrm{C}$, the application of cryo-energy was stopped. Temporal right ventricle pacing was performed during left-sided PV ablation, and continuous phrenic nerve pacing with monitoring of compound motor action potentials was performed during right-sided PV ablation. The application of ablation energy was discontinued to prevent phrenic nerve injury when $>30 \%$ reduction in the amplitude of compound motor action potentials or decreased diaphragmatic excursions were detected. Additional linear ablation at the LA roof and LA posterior wall was attempted using a cryoballoon with a 120-s freezing contiguous fashion protocol between bilateral PV lesions. For touch-up ablation of the PV gap and linear lesion after cryoballoon ablation, additional ablation was performed using an RF catheter (TactiCath, Abbott).

CFRF Ablation The RF ablation procedure was conducted under a low fluoroscopy protocol using an integration system (CARTO UNIVU module, Biosense Webster) fused with intracardiac echocardiography and preprocedural CT images. First, a deflectable sheath (Agilis, Abbott, or Vizigo, Biosense Webster) and a long sheath (Swartz SL0 introducer, Abbott) were inserted into the LA. After an LA CFAE or voltage map was created, bilateral extensive PVI was performed using a CFRF catheter (ThermoCool SmartTouch or ThermoCool SmartTouch SF, Biosense Webster) with the moderate-power (30-35W) 


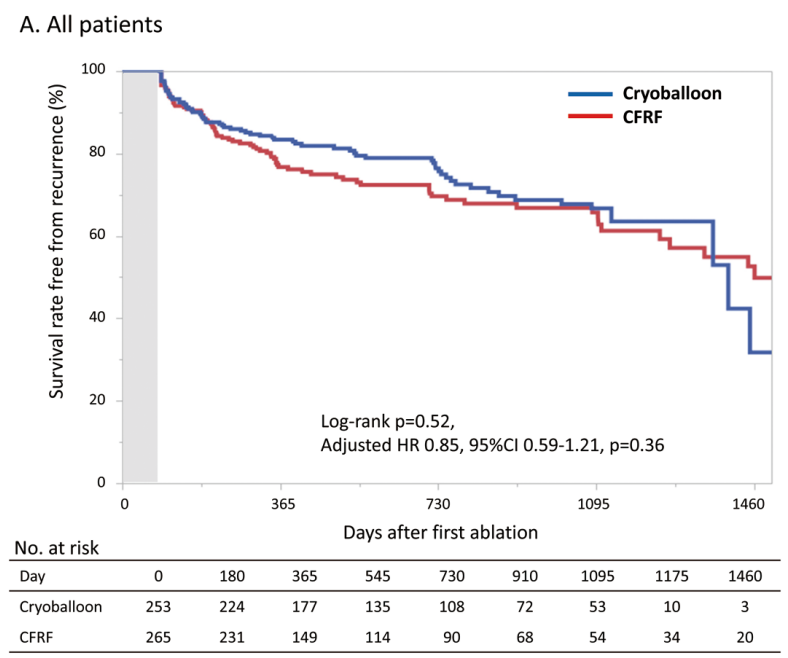

B. PVI alone

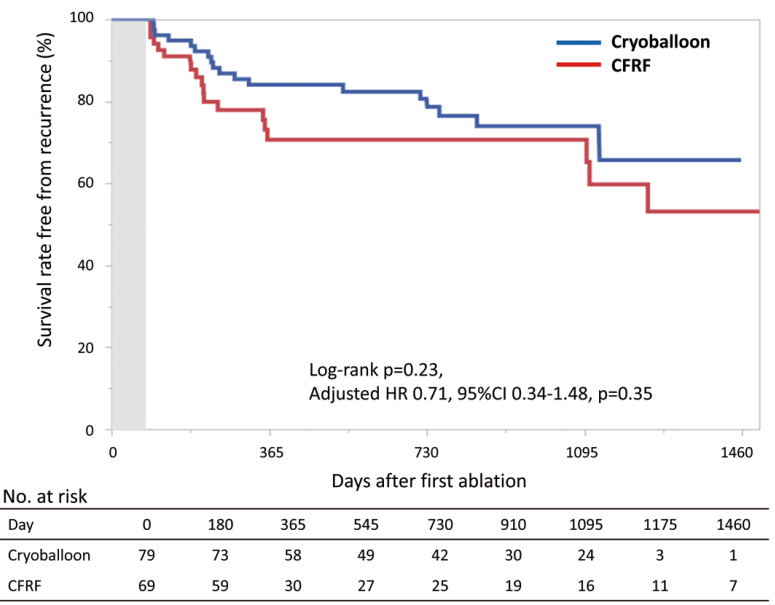

C. PVI plus LA additional ablation

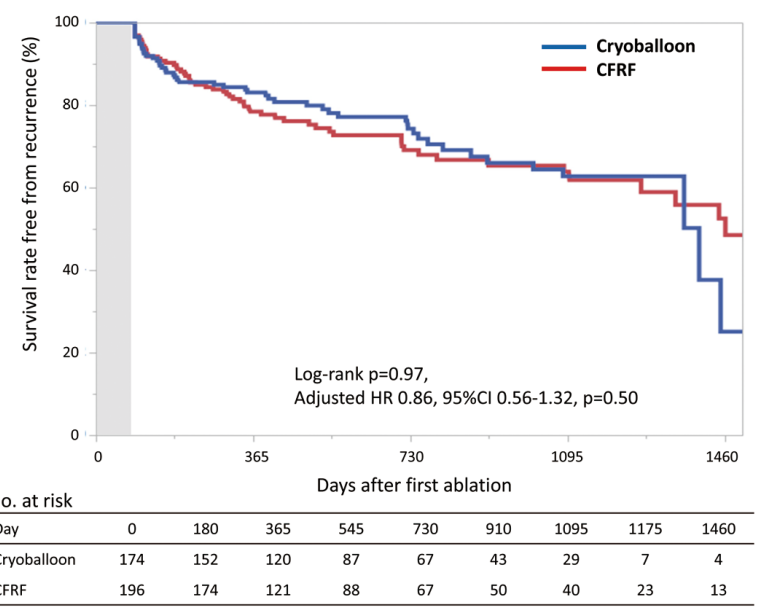

Figure 1. Kaplan-Meier plot of recurrence-free survival rates. Rates of freedom from the primary endpoint (recurrent atrial arrhythmias) after the index ablation after the 3-month blanking period off drugs were not significantly different between the cryoballoon (blue) and CFRF (red) groups in all patients (A), in a subgroup of PVI alone (B), and in a subgroup of PVI plus additional LA ablation strategy $(\mathbf{C})$. CFRF, contact force-sensing radiofrequency ablation; $\mathrm{Cl}$, confidence interval; HR, hazard ratio; LA, left atrium; $\mathrm{PVI}$, pulmonary vein isolation.

or high-power short-duration $(50 \mathrm{~W})$ protocol. The contact force was controlled at $10-20 \mathrm{~g}$. The application time was set for 30 s within a $20-\Omega$ drop of the generator impedance in the condition without lesion formation index or titrated under using the lesion formation index (force-time-index, FTI; or ablation index, AI; Biosense Webster). The lesion formation index targets were set at 400 for FTI and 500 for AI, except along esophageal lesions on the posterior wall $(F T I=300, A I=400)$ with the limitation of esophageal temperature $<41^{\circ} \mathrm{C}$. The ablation lesions were tagged in a contiguous fashion within 6-mm intervals. Realtime monitoring of the PV potentials was performed using a circular mapping catheter. Linear and CFAE ablations were performed using the same application protocol for PVI.

\section{Follow-up}

Scheduled follow-up visits, including a physical examination, 12-lead ECG, and blood examinations, were per- formed at 1, 3, 6, 9, 12, 24, and 36 months after the ablation. Daily pulse self-check or ambulatory ECG monitoring was mandatory, and extra-visit ECG was required when abnormal pulses were detected. The 24-h Holter recordings were performed at 3, 6, 12, 24, and 36 months after the procedure. Cardiac ultrasound examination was performed mandatorily at 6 and 12 months, and if required at 24 and 36 months after the procedure. PV stenosis was assessed using CT imaging 3 months after the procedure.

\section{Endpoints}

The primary endpoint was any recurrence documented as symptomatic or asymptomatic atrial arrhythmia $>30 \mathrm{~s}$ while off antiarrhythmic drugs after the 3-month blanking period. The secondary endpoints included the presence of periprocedural complications (safety assessment) and the need for repeat ablation for recurrent arrhythmias after the index procedure (effectiveness assessment). 


\begin{tabular}{|c|c|c|c|}
\hline & $\begin{array}{c}\text { Cryoballoon } \\
(n=49 / 253)\end{array}$ & $\begin{array}{c}\text { CFRF } \\
(n=49 / 265)\end{array}$ & $P$ value \\
\hline 2nd session, n (\%) & $49(19.4)$ & $49(18.5)$ & 0.80 \\
\hline \multicolumn{4}{|c|}{ Type of recurrent arrhythmia } \\
\hline $\mathrm{AF}, \mathrm{n}(\%)$ & $24(49.0)$ & $26(53.1)$ & \\
\hline AT, n (\%) & $18(36.7)$ & $15(30.6)$ & \\
\hline $\mathrm{AF}+\mathrm{AT}, \mathrm{n}(\%)$ & 7 (14.3) & $8(16.3)$ & \\
\hline \multicolumn{4}{|c|}{ Target for recurrent arrhythmia } \\
\hline Reconducted PV, n (\%) & $14(28.6)$ & $27(55.1)$ & 0.007 \\
\hline SVC, $n(\%)$ & $10(20.4)$ & $9(18.4)$ & 0.80 \\
\hline $\mathrm{RA}, \mathrm{n}(\%)$ & $5(10.2)$ & $9(18.4)$ & 0.25 \\
\hline $\mathrm{LA}, \mathrm{n}(\%)$ & $25(51.0)$ & $12(24.5)$ & 0.006 \\
\hline Reentrant AT, n (\%) & $13(26.5)$ & $17(34.7)$ & 0.38 \\
\hline
\end{tabular}

Continuous variables are shown as mean \pm standard deviation. RA, right atrium. Other abbreviations as in Tables 1,2.

\section{Statistical Analysis}

Baseline patient demographics and procedural and clinical characteristics were compared between cohorts. Continuous variables with normal distribution were confirmed using the Shapiro-Wilk test and compared using the t-test and presented as mean and standard deviation. Non-normally distribution data were compared using the Wilcoxon test and presented as median and interquartile ranges. Fisher's exact test was used to compare the categorical variables. Time-to-event analysis was performed using Kaplan-Meier curves, utilizing the log-rank test and Cox proportional-hazard regression to compare the differences between the groups. Adjusted hazard ratios (HRs) with 95\% confidence intervals (CIs) are shown. For all analyses, $P$ values were two-sided, and statistical significance was set at $\mathrm{P}<0.05$. JMP version 13 (SAS Institute Inc., Cary, NC, USA) was used for all statistical analyses.

\section{Results}

\section{Patients' Characteristics}

The records of 532 consecutive patients who underwent their first CA for persistent AF were reviewed, and 518 patients (cryoballoon: 253; CFRF: 265) were enrolled after exclusion screening. Patients with enlarged LA diameter $(>60 \mathrm{~mm})$ and unstable state of heart failure were excluded (cryoballoon: 3 and 3, respectively; CFRF: 1 and 7, respectively). There were no significant differences in baseline characteristics between the groups, except for a higher proportion of the male sex, longer duration of $\mathrm{AF}$, and less comorbidity of heart failure and valvular heart disease in the cryoballoon group (Table 1). A slightly smaller LA diameter and a greater left ventricular ejection fraction were observed in the echocardiograms in the cryoballoon group compared with the CFRF group.

\section{Ablation Procedures}

In the cryoballoon group, a second-generation catheter was used in 190 patients and a fourth-generation catheter was used in 63 patients. In the CFRF group, a contact force-sensing catheter with a 6-hole irrigation tip was used in 84 patients, and a contact force-sensing catheter with a 56-hole porous tip was used in 181 patients. For the lesion formation index, the FTI and AI were used in 43 and 146 patients, respectively. The moderate-power ablation protocol was used in 140 patients and the high-power short-duration protocol was used in 125 patients. PVI was successfully achieved in all cases, and 3.9\% of the patients in the cryoballoon group required touch-up ablation using an RF catheter (Table 2). The implementation rate of any additional ablation in the LA, including roof line, posterior line, mitral isthmus line, non-PV foci, and defragmentation was comparable (174 [68.8\%] in cryoballoon vs. 196 [74.0\%] in CFRF, $\mathrm{P}=0.19$ ). The success rate of LA roof line and posterior wall isolation was higher in the cryoballoon group, including the concomitant or unaccompanied use of RF catheters (success rate by cryoballoon alone: $89.1 \%$ and $34.2 \%$, respectively). In the CFRF group, the high-power short-duration ablation protocol shortened procedure times compared with the moderate-power ablation protocol (procedure time for PVI: $57.8 \pm 20.3 \mathrm{~min}$ vs. $89.3 \pm 35.6 \mathrm{~min}$; LA dwelling time: $115.1 \pm 33.0 \mathrm{~min}$ vs. $137.9 \pm 41.6 \mathrm{~min}$; total procedure time: $190.9 \pm 40.8 \mathrm{~min}$ vs. 222.0 $\pm 48.5 \mathrm{~min} ; \mathrm{P}<0.0001)$. However, the PVI procedure time, LA dwelling time, and total procedure time with the cryoballoon were shorter in the total cohort, and even in the subgroups of PVI alone or PVI plus LA additional ablation. The fluoroscopy time was longer in the cryoballoon group than in the CFRF group. Periprocedural complications, including cardiac tamponade, worsening of heart failure, and phrenic nerve injury, were similar in both groups.

\section{Clinical Outcomes}

The rate of freedom from the primary endpoint was comparable between groups during the follow-up of $25.5 \pm 12.5$ months $(72.3 \%$ in cryoballoon vs. $69.8 \%$ in CFRF, adjusted HR $0.85,95 \%$ CI $0.59-1.21, \mathrm{P}=0.36$; Figure 1A). In the subgroup analysis of the patients with PVI alone or with PVI plus additional LA ablation, the rates of freedom from atrial arrhythmia recurrence were comparable between the groups (Figure 1B,C). In each treatment group, comparable effects for the primary endpoint were revealed for catheter refinement (fourth/second-generation cryoballoon: adjusted HR $1.45,95 \%$ CI $0.64-3.08, \mathrm{P}=0.36$ ) and advancing protocol (high-power short-duration/moderate-power protocol: adjusted HR $0.77,95 \%$ CI 0.44 1.31, $\mathrm{P}=0.33$ ). During the follow-up period, second repeat ablation sessions for recurrent arrhythmias were comparably performed in each group $(19.4 \%$ in cryoballoon vs. 


\begin{tabular}{|c|c|c|c|c|c|c|c|c|}
\hline \multirow{2}{*}{ Parameter } & \multirow{2}{*}{ Yes, $n$} & \multirow{2}{*}{ No, $n$} & \multicolumn{3}{|c|}{ Univariate analysis } & \multicolumn{3}{|c|}{ Multivariate analysis } \\
\hline & & & HR & $P$ value & $95 \% \mathrm{Cl}$ & HR & $P$ value & $95 \% \mathrm{Cl}$ \\
\hline \multicolumn{9}{|l|}{ Age } \\
\hline$\geq 75$ years & 195 & 323 & 1.16 & 0.37 & $0.83-1.62$ & 0.95 & 0.78 & $0.66-1.36$ \\
\hline \multicolumn{9}{|l|}{ Sex } \\
\hline Male & 383 & 135 & 0.81 & 0.25 & $0.57-1.17$ & 0.89 & 0.56 & $0.61-1.32$ \\
\hline \multicolumn{9}{|l|}{ BMI } \\
\hline$\geq 28$ & 63 & 396 & 0.94 & 0.81 & $0.53-1.54$ & & & \\
\hline$<20$ & 59 & 396 & 1.04 & 0.87 & $0.60-0.69$ & & & \\
\hline \multicolumn{9}{|l|}{ Duration of $\mathrm{AF}$} \\
\hline$>6$ months & 173 & 334 & 0.98 & 0.94 & $0.69-1.39$ & 0.98 & 0.91 & $0.68-1.39$ \\
\hline Heart failure & 148 & 368 & 0.86 & 0.45 & $0.57-1.26$ & 0.82 & 0.32 & $0.54-1.21$ \\
\hline Renal dysfunction & 30 & 482 & 2.03 & 0.03 & $1.06-3.53$ & & & \\
\hline Respiratory disease & 31 & 472 & 0.49 & 0.11 & $0.15-1.15$ & & & \\
\hline Valvular heart disease & 29 & 489 & 0.96 & 0.91 & $0.43-1.83$ & & & \\
\hline \multicolumn{9}{|l|}{ LVEF } \\
\hline$<45 \%$ & 46 & 472 & 0.76 & 0.43 & $0.34-1.45$ & & & \\
\hline \multicolumn{9}{|l|}{ Left atrial diameter } \\
\hline$\geq 50 \mathrm{~mm}$ & 39 & 479 & 0.52 & 0.08 & $0.20-1.08$ & 0.54 & 0.11 & $0.21-1.14$ \\
\hline \multicolumn{9}{|l|}{ Creatinine clearance } \\
\hline$<50 \mathrm{~mL} / \mathrm{min}$ & 76 & 439 & 1.70 & 0.02 & $1.08-2.56$ & 1.91 & 0.007 & $1.19-2.97$ \\
\hline \multicolumn{9}{|l|}{ NT-proBNP } \\
\hline$>1,000 \mathrm{pg} / \mathrm{mL}$ & 198 & 316 & 1.08 & 0.66 & $0.77-1.50$ & & & \\
\hline \multicolumn{9}{|l|}{ LA posterior line } \\
\hline Performed & 311 & 207 & 0.78 & 0.15 & $0.56-1.09$ & & & \\
\hline \multicolumn{9}{|l|}{ LA roof line } \\
\hline Performed & 350 & 168 & 0.79 & 0.17 & $0.56-1.10$ & & & \\
\hline Success & 290 & 168 & 0.74 & 0.10 & $0.52-1.06$ & & & \\
\hline Failure & 60 & 168 & 1.05 & 0.85 & $0.59-1.78$ & & & \\
\hline \multicolumn{9}{|l|}{ LA posterior wall isolation } \\
\hline Performed & 308 & 210 & 0.76 & 0.11 & $0.55-1.06$ & & & \\
\hline Success & 233 & 210 & 0.68 & 0.03 & $0.47-0.97$ & 0.63 & 0.013 & $0.44-0.91$ \\
\hline Failure & 75 & 210 & 1.12 & 0.66 & $0.67-1.78$ & & & \\
\hline \multicolumn{9}{|l|}{ Mitral isthmus line } \\
\hline Performed & 40 & 478 & 1.10 & 0.72 & $0.63-1.82$ & 0.91 & 0.76 & $0.50-1.58$ \\
\hline Success & 33 & 478 & 0.85 & 0.59 & $0.43-1.51$ & & & \\
\hline Failure & 7 & 478 & 3.03 & 0.04 & $1.07-6.67$ & & & \\
\hline \multicolumn{9}{|l|}{ Defragmentation } \\
\hline Performed & 247 & 271 & 1.47 & 0.02 & $1.06-2.05$ & 1.64 & 0.005 & $1.16-2.34$ \\
\hline \multicolumn{9}{|l|}{ Additional LA ablation } \\
\hline Performed & 370 & 148 & 1.21 & 0.32 & $0.84-1.79$ & & & \\
\hline \multicolumn{9}{|l|}{ Catheter selection } \\
\hline Cryoballoon & 253 & 265 & 0.86 & 0.38 & $0.62-1.20$ & 0.85 & 0.36 & $0.59-1.21$ \\
\hline
\end{tabular}

$\mathrm{BMI}$, body mass index; $\mathrm{Cl}$, confidence interval; $\mathrm{HR}$, hazard ratio. Other abbreviations as in Tables 1,2.

$18.5 \%$ in CFRF, $\mathrm{P}=0.80$, Table 3). In the second session, fewer reconducted PVs were observed in the cryoballoon group $(28.6 \%$ in cryoballoon vs. $55.1 \%$ in $\mathrm{CFRF}, \mathrm{P}=0.007)$.

\section{Factors Associated With Efficacy or Safety Endpoints}

With respect to the observed characteristic differences, an adjustment was performed for covariates, including age, sex, duration of AF, heart failure, creatinine clearance, LA size, and additional ablations. Regarding the primary endpoint, univariate and multivariate regression suggested a negative relationship with low creatinine clearance ( $<50 \mathrm{~mL} / \mathrm{min}$, adjusted HR 1.91, 95\% CI 1.19-2.97,
$\mathrm{P}=0.007)$ and the implementation of defragmentation ablation in the LA (adjusted HR 1.64, 95\% CI 1.16-2.34, $\mathrm{P}=0.005$ ), and a positive relationship with successful LA posterior wall isolation (adjusted HR $0.63,95 \%$ CI 0.44 $0.91, \mathrm{P}=0.013$ ) (Table 4). On the other hand, the selection of the cryoballoon catheter was not significantly associated with the primary or secondary endpoints (Figure 2).

\section{Discussion}

We evaluated the effectiveness and safety of cryoballoon ablation for persistent AF compared with CFRF ablation 


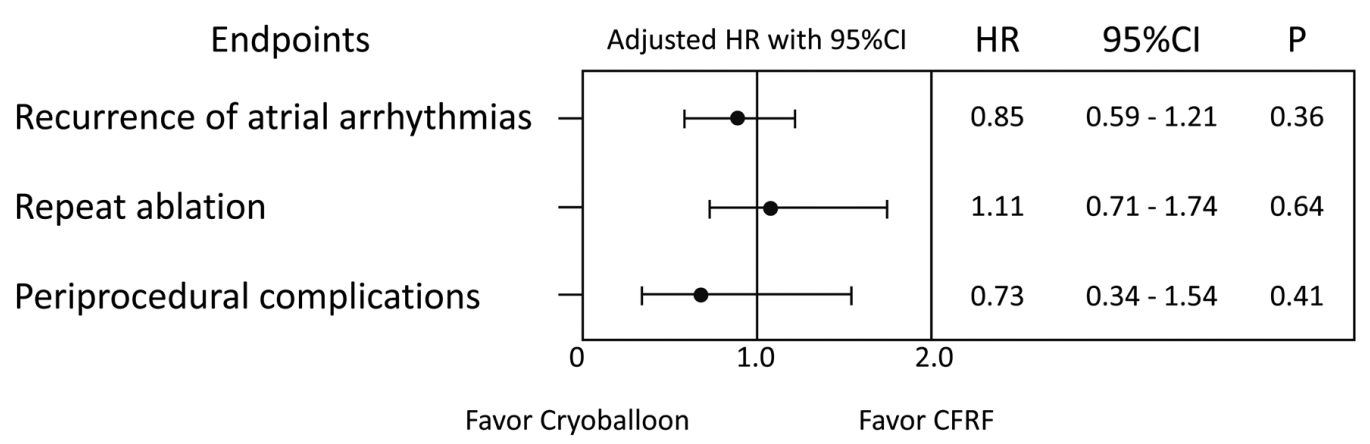

Figure 2. Proportional-hazards model assessment for the endpoints. The adjusted hazard ratios of the multivariable models with confidence intervals and the $\mathrm{P}$ values for cryoballoon vs. CFRF after the index ablation are shown. CFRF, contact force-sensing radiofrequency ablation; $\mathrm{Cl}$, confidence interval; $\mathrm{HR}$, hazard ratio.

in a real-world clinical setting. To our knowledge, this is the first comparative observational study focusing on the assessment of cryoballoon ablation for persistent AF in an Asian population using both contemporary advanced catheters and ablation protocols. We found comparable effectiveness and safety of cryoballoon and CFRF ablation for persistent AF, even in the subgroups of PVI alone or PVI plus additional LA ablation if patients were selected based on CT images, and patients with a thin LA or deformed PV were excluded from cryoballoon use. The primary endpoint (atrial arrhythmia recurrence) was not associated with the use of a cryoballoon catheter in the hazard regression analysis. The secondary safety endpoints (periprocedural complications and the need for repeat ablation) were also comparable between cryoballoon and CFRF ablation.

\section{Efficacy Outcomes}

The results of this study showed comparable effectiveness for the primary endpoint between cryoballoon and CFRF ablation for persistent AF. Although previous comparative studies of the effectiveness of cryoballoon and RF ablation have been conducted, contemporary devices including the fourth-generation cryoballoon and advanced protocols including high-power short-duration guided by the AI have not been used. ${ }^{\mathbf{1 2 , 1 7 , 1 9 , 2 0}}$ In that respect, this study was able to compare widely used and highly reliable devices, and we consider that the therapeutic effect on persistent AF in current clinical practice was clearly shown. However, this study was not a prospective allocation study, and there were significant differences in the characteristics of the 2 groups. In addition, although the cryoballoon is designed for PVI and can provide effective therapeutic effects, the requirement for additional LA ablations may have influenced patient selection and treatment strategy. For these reasons, a subgroup analysis was conducted, but no effect of catheter selection on the primary endpoint was observed with PVI alone or with PVI plus additional ablation. Furthermore, no effect of catheter selection was observed in the analysis with full adjustment for the observed differences. These findings indicated that the clinical effect of the cryoballoon in patients with persistent AF was shown not only in the PVI alone strategy, which has been reported, ${ }^{5-11}$ but also in PVI plus additional treatment in line with actual clinical practice. ${ }^{\mathbf{1 7}, 20}$

The rate of repeat ablation for recurrent arrhythmias was similar in both groups. This finding was different from previous reports, which showed low repeat ablation rates in cryoballoon ablation for paroxysmal AF. ${ }^{14}$ However, the durability of PVI at the repeat session was significantly higher in the cryoballoon group, as in previous reports. ${ }^{\mathbf{1 4}, 15}$ Therefore, cryoballoon ablation can be expected to have high PVI durability even in persistent AF, but it is considered that the cause of clinical recurrence in persistent AF is largely influenced by factors other than PVI reconduction. These findings are consistent with a previous report that presented different results between paroxysmal AF and persistent AF. ${ }^{19}$

\section{Procedure Time}

The procedure time for cryoballoon ablation was notably shorter than that for CFRF ablation. This study showed not only the effect of frequent additional LA treatment in the CFRF group, but also fully demonstrated the advantage of the one-shot PVI with the cryoballoon over the point-by-point application of CFRF. In CFRF, the advanced high-power short-duration protocol has shortened the procedure time; however, the advantage of the cryoballoon remained. Nevertheless, fluoroscopy time was significantly longer with the cryoballoon, and reducing this is an issue.

\section{Safety Outcomes}

In this study, the incidence of complications in both groups was similar, and both modalities were considered safe, comparable to previous reports. ${ }^{16,21}$ There was also no significant difference in the incidence of individual complications between the two groups. Phrenic nerve palsy has been reported as a characteristic complication of cryoballoon ablation, but the occurrence and aggravation can be prevented and reduced by the application of sufficient precautions. ${ }^{22}$ On the other hand, the exacerbation of heart failure, a characteristic complication of irrigation catheter use, seems to have been reduced by the new high-power short-duration protocol.

Catheter selection was not associated with the safetyfocused secondary endpoint (periprocedural complications) as noted in previous reports. ${ }^{22}$ 


\section{Additional Ablation Strategy}

In this study, additional LA ablations were at the operator's discretion; thus, a large proportion of patients in both groups underwent optional therapies beyond PVI, including LA roof line, LA posterior line, LA posterior wall isolation by the double LA line, non-PV foci, and defragmentation. The high durability of the cryoballoon was expected to improve outcomes, including post-PVI strategy, but additional LA ablation did not affect the primary endpoint as noted in a previous RF ablation report. ${ }^{18}$ In the multivariate hazard analysis, isolation of the posterior wall of the LA had favorable effects on the primary endpoint; however, defragmentation worsened the outcome. Therefore, the effect of additional LA posterior wall isolation may have been offset by LA defragmentation. The addition of LA posterior wall isolation with cryoballoon therapy has previously been reported to improve clinical outcomes. $^{23,24}$ The LA roofline success rate with the cryoballoon has been reported to be sufficiently high, ${ }^{25,26}$ as was the case in this study. On the other hand, the success rate of the LA posterior line was low, and in many cases in the cryoballoon group, LA posterior line creation with RF alone or touch-up with RF was performed. This study revealed that this strategy of LA posterior wall isolation, including the combined use of RF, was also effective with cryoballoon therapy; however, there are reports that complete isolation of the LA posterior wall is unnecessary. ${ }^{20,27}$ Therefore, a prospective large-scale verification is expected.

\section{Study Limitations}

Several limitations of our study caused by the observational study design should be noted. First, treatment assignment was not randomized, and the strategy was at the operators' discretion. However, consecutive patients were enrolled, and clinical features were similar in both groups, except for some characteristics. All baseline characteristics between the groups were revealed not to affect the endpoints. On the other hand, the effect of the cryoballoon on excluded patients cannot be clarified in this study. Although all patients in the cryoballoon group were treated using cryoballoon alone in principle, some required strategies including PVI touch-up and additional treatment were performed in combination with CFRF. These concomitant uses were considered to have an effect on the results and evaluation. It would be useful to investigate these concomitant uses in actual clinical practice. However, these concomitant uses increase the cost of treatment and may not be available in some regions. The recurrence of asymptomatic arrhythmia may have been underestimated because of the limited assessment with regular ECG and Holter monitoring ECG and not long-term monitoring. Therefore, multicenter controlled studies with a larger patient population and longterm ECG monitoring are required.

\section{Conclusions}

With proper patient selection based on preprocedural anatomical images, contemporary cryoballoon ablation provided acceptable effectiveness and safety compared with advanced CFRF ablation, including the high-power shortduration protocol guided by the AI, in Japanese patients with persistent $\mathrm{AF}$ in current clinical practice. The incidence of repeat ablation was comparable to that of CFRF therapy, but the durability of PVI with cryoballoon therapy was higher than that in CFRF ablation.

\section{Acknowledgments}

We thank the Center for Clinical Research and Innovation (CCRI) of Kobe City Medical Center General Hospital for their advice, and Editage (www.editage.com) for English language editing.

\section{Conflict of Interest}

A.K. has received lecture fees from Medtronic Japan Inc. All other authors have no conflicts of interest to declare.

\section{Relationships With Industry}

The relationship with industry mentioned above presents no conflicts of interest regarding this study.

\section{IRB Information}

The study was approved by the Institutional Review Board of Kobe City Medical Center General Hospital (reference no. zn210716).

\section{Data Availability}

The deidentified participant data will not be shared, except for a considerable useful request.

\section{References}

1. Inoue H, Fujiki A, Origasa H, Ogawa S, Okumura K, Kubota I, et al. Prevalence of atrial fibrillation in the general population of Japan: An analysis based on periodic health examination. Int $J$ Cardiol 2009; 137: 102-107.

2. Haïssaguerre M, Jaïs P, Shah DC, Takahashi A, Hocini M, Quiniou G, et al. Spontaneous initiation of atrial fibrillation by ectopic beats originating in the pulmonary veins. $N$ Engl J Med 1998; 339: 659-666.

3. Calkins H, Hindricks G, Cappato R, Kim YH, Saad EB, Aguinaga L, et al. 2017 HRS/EHRA/ECAS/APHRS/SOLAECE expert consensus statement on catheter and surgical ablation of atrial fibrillation. J Arrhythmia 2017; 33: 369-409.

4. Kobori A, Sasaki Y, Pak M, Okada T, Toyota T, Kim K, et al. Early experiences with three types of balloon-based ablation catheters in patients with paroxysmal atrial fibrillation. Heart Rhythm O2 2021; 2: 223-230.

5. Su W, Reddy V, Bhasin K, Champagne J, Sangrigoli R, Braegelmann $\mathrm{K}$, et al. Cryoballoon ablation of pulmonary veins for persistent atrial fibrillation: Results from the multicenter STOP Persistent AF trial. Heart Rhythm 2020; 17: $1841-1847$.

6. Tondo C, Iacopino S, Pieragnoli P, Molon G, Verlato R, Curnis A, et al. Pulmonary vein isolation cryoablation for patients with persistent and long-standing persistent atrial fibrillation: Clinical outcomes from the real-world multicenter observational project. Heart Rhythm 2018; 15: 363-368.

7. Lemes C, Wissner E, Lin T, Mathew S, Deiss S, Rillig A, et al. One-year clinical outcome after pulmonary vein isolation in persistent atrial fibrillation using the second-generation $28 \mathrm{~mm}$ cryoballoon: A retrospective analysis. Europace 2016; 18: 201-205.

8. Straube F, Hartl S, Dorwarth U, Wankerl M, Bunz B, Ebersberger $\mathrm{U}$, et al. Cryoballoon ablation for persistent atrial fibrillation: Large single-center experience. J Cardiol 2016; 68: 492-497.

9. Tscholl V, Lsharaf AK, Lin T, Bellmann B, Biewener S, Nagel $\mathrm{P}$, et al. Two years outcome in patients with persistent atrial fibrillation after pulmonary vein isolation using the second-generation 28-mm cryoballoon. Heart Rhythm 2016; 13: 1817-1822.

10. Koektuerk B, Yorgun H, Hengeoez O, Turan CH, Dahmen A, Yang A, et al. Cryoballoon ablation for pulmonary vein isolation in patients with persistent atrial fibrillation: One-year outcome using second generation cryoballoon. Circ Arrhythm Electrophysiol 2015; 8: 1073-1079.

11. Ciconte G, Ottaviano L, de Asmundis C, Baltogiannis G, Conte $\mathrm{G}$, Sieira $\mathbf{J}$, et al. Pulmonary vein isolation as index procedure for persistent atrial fibrillation: One-year clinical outcome after ablation using the second-generation cryoballoon. Heart Rhythm 2015; 12: 60-66.

12. Ciconte G, Baltogiannis G, de Asmundis C, Sieira J, Conte G, Di Giovanni G, et al. Circumferential pulmonary vein isolation as index procedure for persistent atrial fibrillation: A comparison between radiofrequency catheter ablation and second generation cryoballoon ablation. Europace 2015; 17: 559-565.

13. Kuck KH, Brugada J, Furnkranz A, Metzner A, Ouyang F, 
Chun KR, et al. Cryoballoon or radiofrequency ablation for paroxysmal atrial fibrillation. $N$ Engl J Med 2016; 374: 2235-2245.

14. Kuck KH, Furnkranz A, Chun KR, Metzner A, Ouyang F, Schluter M, et al. Cryoballoon or radiofrequency ablation for symptomatic paroxysmal atrial fibrillation: Reintervention, rehospitalization, and quality-of-life outcomes in the FIRE AND ICE trial. Eur Heart J 2016; 37: 2858-2865.

15. Ciconte G, Velagić V, Mugnai G, Saitoh Y, Irfan G, Hunuk B, et al. Electrophysiological findings following pulmonary vein isolation using radiofrequency catheter guided by contact-force and second-generation cryoballoon: Lessons from repeat ablation procedures. Europace 2016; 18: 71-77.

16. Winkle RA, Mohanty S, Patrawala RA, Mead RH, Kong MH, Engel G, et al. Low complication rates using high power (45$50 \mathrm{~W}$ ) for short duration for atrial fibrillation ablations. Heart Rhythm 2019; 16: 165-169.

17. Bisignani A, Cecchini F, Mugnai G, Overeinder I, Sieira J, Osório TG, et al. Single procedural outcomes in the setting of percutaneous ablation for persistent atrial fibrillation: A propensity-matched score comparison between different strategies. $J$ Interv Card Electrophysiol, doi:10.1007/s10840-021-00968-2.

18. Verma A, Jiang CY, Betts TR, Chen J, Deisenhofer I, Mantovan $\mathrm{R}$, et al; STAR AF II Investigators. Approaches to catheter ablation for persistent atrial fibrillation. N Engl J Med 2015; 372: $1812-1822$.

19. Hoffmann E, Straube F, Wegscheider K, Kuniss M, Andresen $\mathrm{D}, \mathrm{Wu} \mathrm{LQ}$, et al. Outcomes of cryoballoon or radiofrequency ablation in symptomatic paroxysmal or persistent atrial fibrillation. Europace 2019; 21: 1313-1324.

20. Yokokawa M, Chugh A, Latchamsetty R, Ghanbari H, Crawford $\mathrm{T}$, Jongnarangsin $\mathrm{K}$, et al. Cryoballoon antral pulmonary vein isolation vs. contact force-sensing radiofrequency catheter ablation for pulmonary vein and posterior left atrial isolation in patients with persistent atrial fibrillation. Heart Rhythm 2018; 15: $1835-1841$.
21. Miyazaki S, Kobori A, Sasaki Y, Miyamoto K, Sato E, Hanazawa K, et al. Real-world safety profile of atrial fibrillation ablation using a second-generation cryoballoon in Japan: Insight from a large multicenter observational study. JACC Clin Electrophysiol 2021; 7: 604-613.

22. Steinbeck G, Sinner MF, Lutz M, Müller-Nurasyid M, Kääb S, Reinecke H. Incidence of complications related to catheter ablation of atrial fibrillation and atrial flutter: A nationwide in-hospital analysis of administrative data for Germany in 2014. Eur Heart J 2018; 39: 4020-4029.

23. Shao M, Shang L, Shi J, Zhao Y, Zhang W, Zhang L, et al. The safety and efficacy of second-generation cryoballoon ablation plus catheter ablation for persistent atrial fibrillation: A systematic review and meta-analysis. PLoS One 2018; 13: e0206362.

24. Aryana A, Baker JH, Espinosa Ginic MA, Pujara DK, Bowers MR, O'Neill PG, et al. Posterior wall isolation using the cryoballoon in conjunction with pulmonary vein ablation is superior to pulmonary vein isolation alone in patients with persistent atrial fibrillation: A multicenter experience. Heart Rhythm 2018; 15: $1121-1129$

25. Nishimura T, Yamauchi Y, Aoyagi H, Tsuchiya Y, Shigeta T, Nakamura R, et al. The clinical impact of the left atrial posterior wall lesion formation by the cryoballoon application for persistent atrial fibrillation: Feasibility and clinical implications. $J$ Cardiovasc Electrophysiol 2019; 30: 805-814.

26. Kuniss M, Greiß H, Pajitnev D, Akkaya E, Deubner N, Hain A, et al. Cryoballoon ablation of persistent atrial fibrillation: feasibility and safety of left atrial roof ablation with generation of conduction block in addition to antral pulmonary vein isolation. Europace 2017; 19: 1109-1115.

27. Nanbu T, Yotsukura A, Suzuki G, Ishidoya Y, Sano F, Yoshida I, et al. Important factors in left atrial posterior wall isolation using 28-mm cryoballoon ablation for persistent atrial fibrillation-Block line or isolation area? J Cardiovasc Electrophysiol 2020; 31: 119-127. 